\title{
The Mechanism of Cosmic-Ray Counter Action
}

\author{
Carl D. Anderson, R. A. Millikan, Seth Neddermeyer and William Pickering, Norman Bridge Laboratory of
} Physics, California Institute of Technology

(Received December 26, 1933)

\begin{abstract}
A study of photographs taken in a very powerful magnetic field with the aid of a cloud chamber activated by counter responses shows (1) that such an arrangement has a strong selective action on showers; (2) that showers so selected have an exceptional multiplicity of tracks; (3) that the two counters show simultaneous responses when no single particle can pass through both of them; (4) that in general these showers consist of a mixture of positive and negative electrons rather than of electrons and protons, no effects certainly attributable to neutrons
\end{abstract}

being observed; (5) that these electrons in getting out of the nucleus some times produce an intense photon spray of the nature of "brems strahlung"; (6) that these photon sprays are increasingly responsible for the simultaneous activation of the two counters the thicker the intervening lead; (7) that a light element like carbon has little effect in producing either showers or sprays; (8) that the total energy of a shower is not larger than that of single electrons $(+$ or -$)$; (9) that the Dirac theory encounters certain difficulties in accounting for the observed effects.

\section{Historical}

$\mathrm{A}^{\mathrm{s}}$ pointed out by Millikan ${ }^{1}$ in 1932, coincidences in the responses of two counters placed on a vertical line and separated by considerable thicknesses of heavy matter, such, for example as a meter of lead, cannot in general be due to the passage of one charged particle through both counters and the intervening lead, but must rather be due to some mechanism by which a photon can release successively along, or in the general neighborhood of, its path a number of different particles whose separate but practically simultaneous action on the two or more counters is responsible for the observed coincidences. The evidence advanced for this view was as follows: Anderson, Millikan and Neddermeyer had measured (1) the actual energy distribution among the particles whose passage through a gas-filled chamber is responsible for practically all the ionization therein produced, and had found that 75 percent of these particles have energies under a billion $\left(10^{9}\right)$ volts, while a negligible fraction have energies as high as $3 \times 10^{9}$ volts. $^{2}$ They had (2) measured directly the energy loss of these particles in passing through lead and had found it about 35 million volts per $\mathrm{cm}$ of $\mathrm{Pb}^{2}$. This meant

${ }^{1}$ R. A. Millikan, Phys. Rev. 43, 661 (1933); Institut Poincarè Proceedings (1933).

${ }^{2}$ C. D. Anderson, Phys. Rev. 44, 406 (1933); Millikan and Anderson, Phys. Rev. 40, 325 (1932). that a billion volt particle could not pass through more than $30 \mathrm{~cm}$ of lead and that practically no particles of those existing at sea level could pass through a meter of lead. (3) Guided by these facts Pickering had actually changed the number of coincidences observed in three counters without changing the intervening lead, but rather by merely changing the distribution of lead about the line joining the three counters. He had also shown that with large quantities of lead intervening between both the upper and lower pairs of the three counters displacing the counters from a straight line did not necessarily reduce the number of coincidences.

The present experiments not only confirm the foregoing viewpoint, but they show clearly what is the actual mechanism of counter action when considerable amounts of heavy material intervene between the counters. In order to appreciate the nature of the evidence the following previously established facts, obtained by the method of random exposure, must be borne in mind.

\section{Summary of Results with Random Cloud- Chamber Expansions}

In all our preceding measurements on the energies of the cosmic-ray ionizing particles, we have obtained about one good track for every thirty exposures, but since an exposure can be made about once every ten seconds, a successful 
exposure can be obtained about once every five minutes. This is more rapidly than the tracks can be measured and analyzed any way, and the motion picture film used is inexpensive so that for rapidity of work there was no advantage in arranging to have the passage of the rays themselves produce the cloud-chamber expansions and the photographic exposures. Further, for the accurate measurement of cosmic-ray energies the random method has very great advantages. For when the passage of the particle itself precedes the expansion, as it obviously must do if it produces it, this expansion may, and often does, seriously distort the track, but when the random method is used the sharpest tracks are obtained, and without distortion, when the particle passes at the instant at which the expansion is completed or very shortly thereafter. Out of a group of 815 cosmic-ray photographs taken by this method on which a good track or tracks were obtained, about 708 , or 88 percent, corresponded to single isolated tracks; 82 , or 10 percent, corresponded to double tracks or pairs; 7 photographs showed three tracks, i.e., two positives and a negative or two negatives and a positive; 7 photographs showed four tracks; 3 showed 5 tracks; while 7 showed between 5 and 10 tracks; all the tracks on a given photograph being associated in a sense that they are produced by particles passing through the chamber at the same instant as revealed by the identity in sharpness or diffuseness of them all, but not always emanating from a single center; and finally, but one photograph out of the 815 revealed more than 10 tracks. In other words, 88 percent of all the photographs reveal single "electron-shots," while 12 percent reveal "showers," a shower being defined as two or more tracks associated in time. So far as we can now see, from our energy measurements in our powerful magnetic field as well as from our ion-counts, all the electron shots are due to free positive electrons (positrons), or to free negative electrons (negatrons), ${ }^{3}$ practically

\footnotetext{
${ }^{3}$ To remove the ambiguity in the definition of the term "electron" existing at the present time because of the double sense in which it is used in the literature, namely, to denote on the one hand-as for example in the universally used expression electron-volts - the magnitude of the elementary quantity of electric charge, and on the other hand, the name of a particle of a particular mass, the terms "negatron" and "positron" are here used. These
}

none to protons. As to energies, a third of the tracks taken at sea level show energies under 350 million volts, 75 percent show energies under a billion volts, while 98 percent show energies under 3 billion volts, the highest energies so far obtained being shown by single tracks, not by the summation of the energies in a shower.

Practically all of the showers are certainly due to photon encounters with an atomic nucleus for though we have had a bar of lead, a centimeter thick, across the middle of the cloud chamber, and have observed the tracks of a great number of free electrons, both positive and negative, passing through this lead, only in two cases out of say a thousand, have we seen a pair of tracks one of which was a positron produced from the passage of an electron, positive or negative, through the lead. This kind of an encounterthat of an electron with a nucleus, yielding a positron or a pair-is then a very rare event, while in a great many cases a pair emerges from the lower side of the lead when no ionizing ray is seen entering the upper side. This must, so far as we can see, be the result of the encounter of a photon with a nucleus.

\section{Geiger-Counter Controlled Exposures}

Having obtained, from our method of photographing random cosmic-ray shots, the foregoing statistical information as to the normal behaviors of cosmic-ray nuclear encounters and normal cosmic secondary particles, and having deduced

terms are used merely as convenient contractions for the fully descriptive particle designations, "free negative electron" and "free positive electron." The term electron then retains its historical, derivative, and logical meaning as the name of the elementary unit of charge, and the present ambiguity no longer remains. It is pointed out that this suggestion is not at all in conflict with the tradition and usage of the term electron. Even today probably nine-tenths of the usage has reference in the mind of the author to charge rather than to mass, as to take but a single example, in all cases in which the number of electrons going to a given electrode is under consideration. The usage we are suggesting is merely for the sake of removing the ambiguity, the bad effects of which are becoming increasingly felt since the discovery of the "free positive electron," and since the discussion of nuclear processes has become more common. In this usage there is no difficulty in speaking of electrons as existing in the nucleus since one has then in mind only the number of units of electric charge. 
therefrom the foregoing theory as to the nature of cosmic-ray counter action, it was clear that if our reasoning were correct, the use of a pair of counters above and below a cloud chamber for the purpose of letting the simultaneous response of these two counters activate a trip, which, in turn, produced the expansion and took the photograph. would not only throw new light on the mechanism of counter action, but would select showers for special study in our 20,000 gauss field. For Anderson ${ }^{4}$ had published in August, 1932 photographs which showed a group of tracks associated in time, not all of which emanated from the same center, and therefore, which must have involved some non-ionizing mechanism to connect the tracks so associated. Blackett and Occhialini's photographs (March, 1933) present similar evidence. ${ }^{5}$ These observers had not, however, a sufficiently strong magnetic field to measure the total energies of their showers, nor to differentiate in many cases positive and negative electron tracks, and this measurement and differentiation are vital for some of the conclusions at which we arrive herewith.

A cloud chamber actuated by tube-counters is effective only if the time-lag between the passage of the particle through the counters and the completion of the expansion is made extremely small, so that only a negligible diffusion of the ions along the path of the particle occurs before the vapor-track is formed.

f Our apparatus was designed for particularly rapid operation, and the tracks obtained are comparable in sharpness with the very sharpest obtained by the random method of photographing. The apparatus is wholly self-operating, all the resetting operations being made automatically, placing the apparatus in a sensitive state 14 seconds after an exposure. The tube-counters and the vacuum-tube circuit for selecting the simultaneous responses of the two counters situated just above and below the expansion chamber were built, along new lines too, by one of us, Mr. Pickering. Just above the upper counter is placed a bar of lead about $1 \times 1$ inch in cross section (the same as that of the counters) so as to

\footnotetext{
${ }^{4}$ Anderson, Phys. Rev. 41, 409 (1932).

${ }^{5}$ Blackett and Occhialini, Proc. Roy. Soc. A139, 699 (1933).
}

facilitate photon-encounters with the nuclei of lead atoms. Secondary electron-shots resulting from such encounters, if they pass through both counters and thus produce simultaneous counter responses, must also pass through the expansion chamber and appear there as a track in the plane defined by these two counters.

\section{Results Obtained with Geiger Counter Controlled Exposures}

Figs. 1 to 15 show photographs obtained with Geiger counter controlled cloud chamber. The effective diameter of the chamber is $14 \mathrm{~cm}$ and its effective depth $1.5 \mathrm{~cm}$. Two views of the chamber are shown; in each case the one on the left is the direct central view, the right-hand one a mirror image of the central view. It is thus possible to view the tracks stereoscopically and reproduce the effects in space.

Figs. 1 to 6 and 9 to 14 show a plate of lead $1 \mathrm{~cm}$ thick across the center of the chamber. In Fig. 15 a plate of graphite of $1.4 \mathrm{~cm}$ thickness replaces the lead. A plate of lead of $2 \mathrm{~mm}$ thickness across the upper part of the chamber was present in all cases. In the left-hand or direct view particles of positive charge are evidenced by a counter-clockwise sense of rotation in the magnetic field.

(1) The first result appearing from our photographs thus taken, is that as earlier suggested by Blackett and Occhialini this arrangement has a very marked selective action on showers, since these appear very much more frequently than in photographs taken with the aid of the random method described above.

(2) The second result is that the showers caught with the present arrangement show in many cases very many more tracks than do the showers heretofore photographed. This again shows the selectivity, for showers, of this method.

(3) The third result is that not infrequently the two counters show simultaneous responses when no electron shot appears which can possibly pass through both counters and the expansion chamber. This behavior is very fully shown in photographs 1 to 6 , and 10,11 and 15 (see also legends). These photographs are all illustrations of the situation in which the counters were clearly set off, not by a single particle going through both counters. but, 


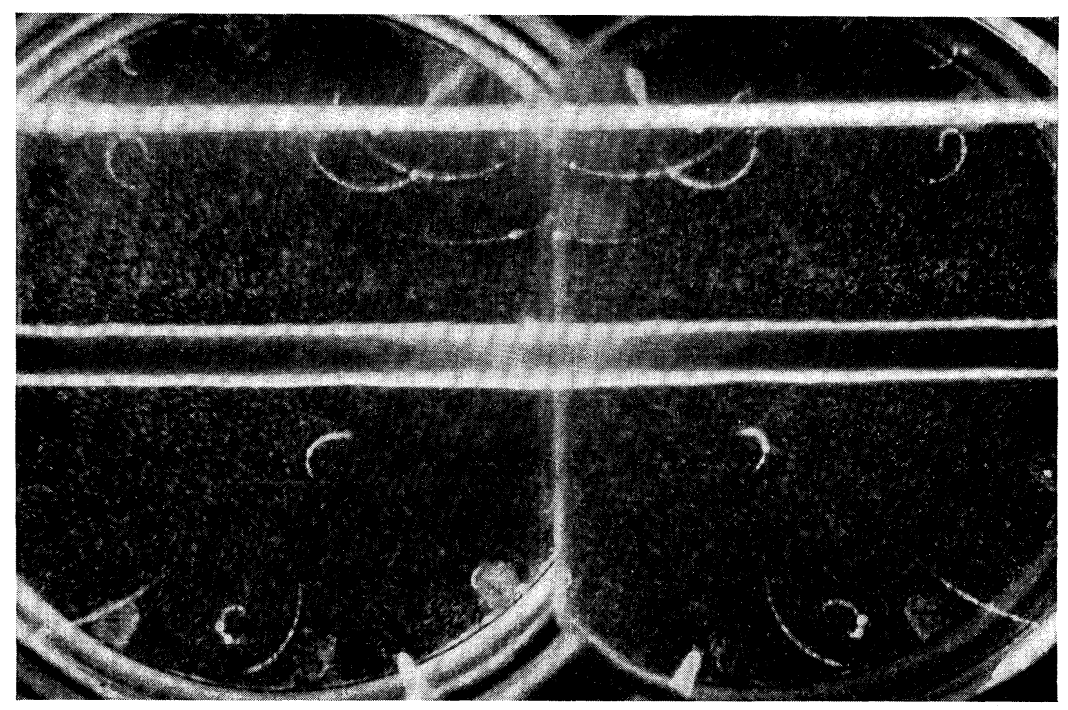

FIG. 1. 17,000 gauss field. The left-hand exposure is the direct photograph, the other the reflection taken for stereoscopic purposes. Six low energy electrons are seen between the two lead plates and four below the lower one. Stereoscopic vision shows that eight of these ten come from the wall and two from the upper lead plate. The short, very heavy track above the top plate, probably not associated in time with the others, may be a recoil nucleus.

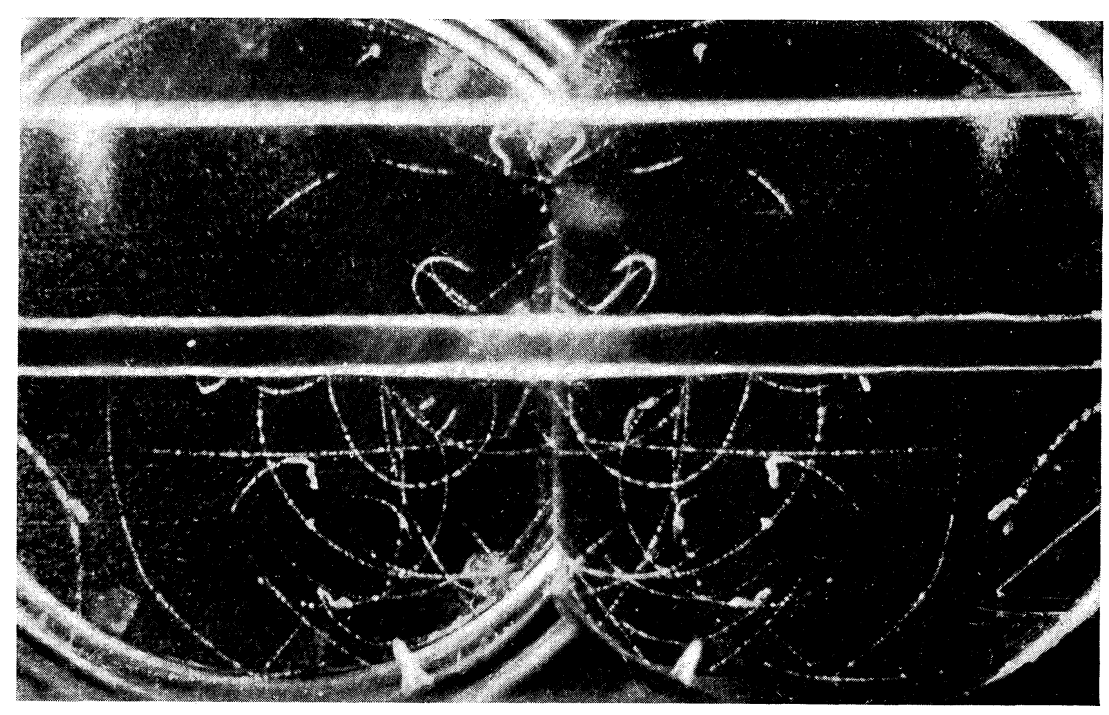

FIG. 2. 825 gauss field. This photograph shows eight electron tracks between the two plates and 15-20 below the central one, mostly of the order of but a million electron-volts. Stereoscopic vision shows two pairs produced within the central plate and emerging from its lower face. Most of the remaining electrons come from the walls. Their distribution in direction is extremely various, indicating that they arise from the absorption of a spray of photons, probably originating in the lead bar above the upper counter. The horizontal track cutting across all the others is particularly interesting. 


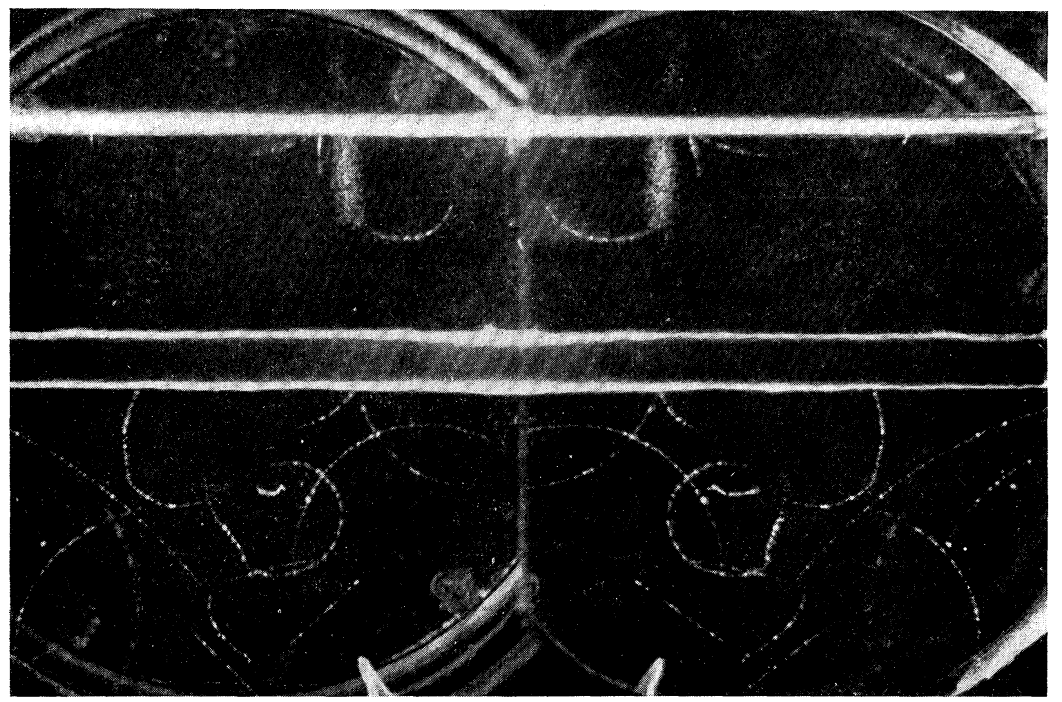

FIG. 3. 825 gauss field. A pair ejected from the upper lead plate and another from the central lead plate; also a group of some fifteen tracks of irregular distribution all ejected by soft photon rays, probably originating in the lead bar above the upper counter.
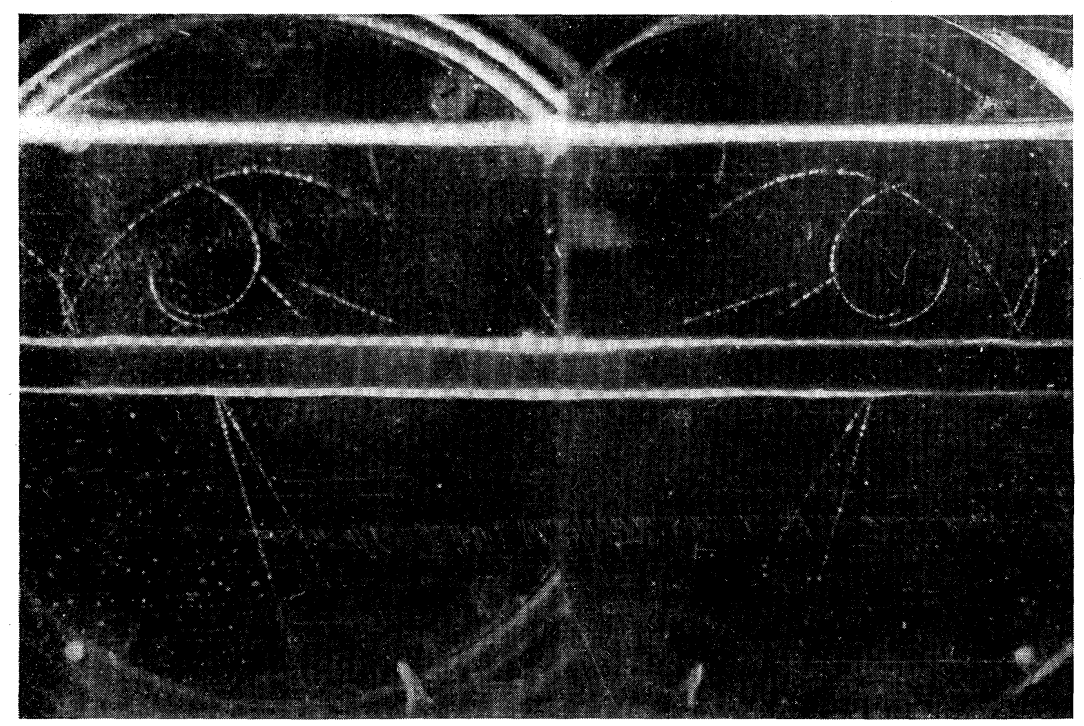

FIG. 4. 825 gauss field. A rather high energy pair is ejected from the lead plate by the absorption within it of a photon. The signs and energies of the components cannot be determined because of the weakness of the field. 

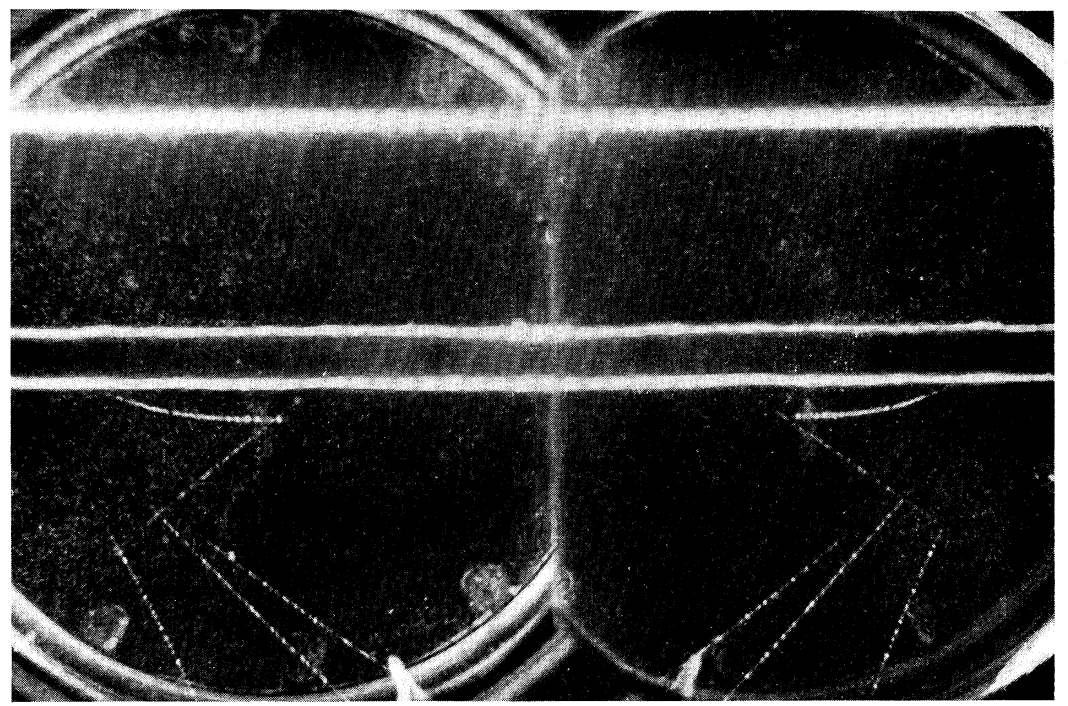

Fig. 5. 17,000 gauss field. Four tracks come from a common region behind the chamber. The fifth is definitely associated with them in time but has a totally different origin. We again postulate a secondary photon spray.

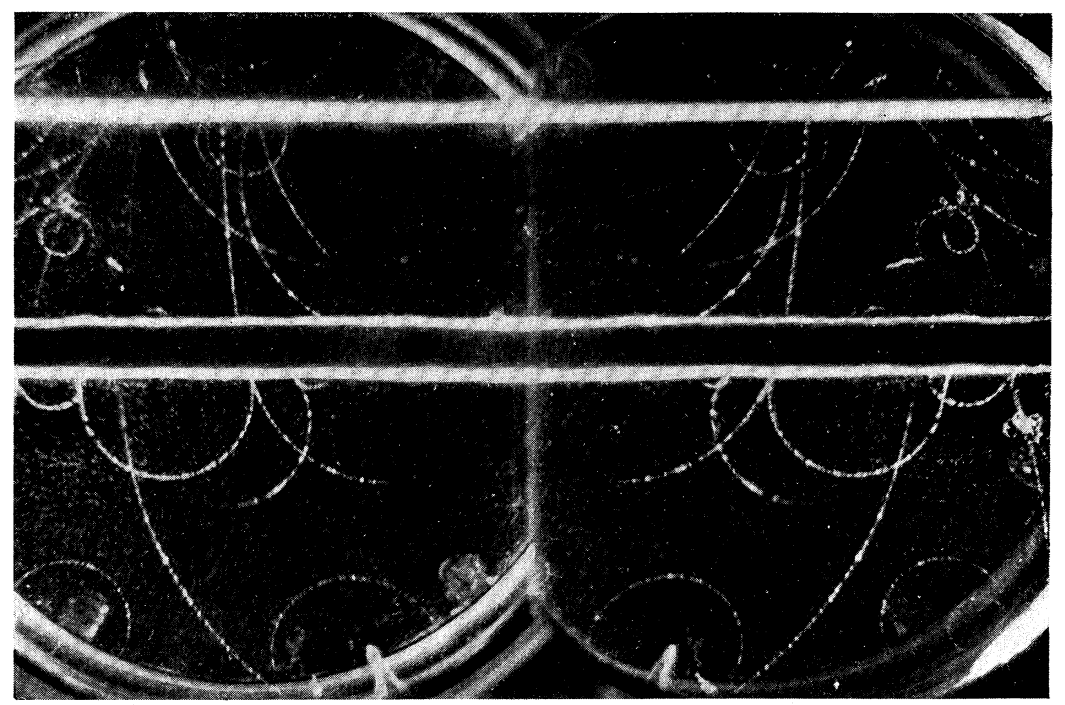

FIG. 6. 17,000 gauss field. Here again no single particle goes through the counters and the cloud chamber, but a secondary photon spray originating above ejects from the lead plates and the walls several pairs and a number of single electrons. A positron of energy $140 \times 10^{6}$ electronvolts penetrates the central $1 \mathrm{~cm}$ lead plate and emerges with an energy of $13 \times 10^{6}$ electronvolts after giving by a close encounter $6.7 \times 10^{6}$ electron-volts to a negatron. 


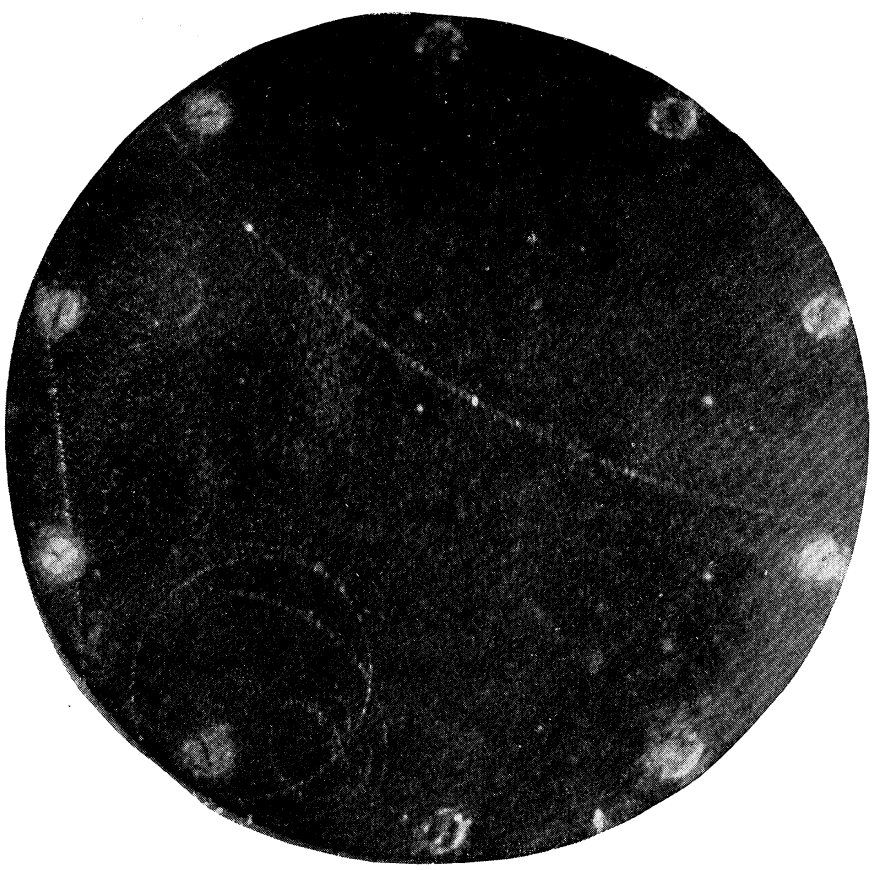

FIG. 7. 17,000 gauss field. An electron pair, positron 75 million electron-volts, negatron 290 million ejected presumably from the nucleus of a lead atom above the upper counter. These electrons in getting through or out of the nucleus presumably collided with its mass, and produced thereby "brems-strahlung." This photon spray shot downward and its absorption in the gas of the chamber, or the surface layer of its wall, produced the four secondaries seen between the electron tracks of energies in millions of electron-volts $9,9,4,1$.

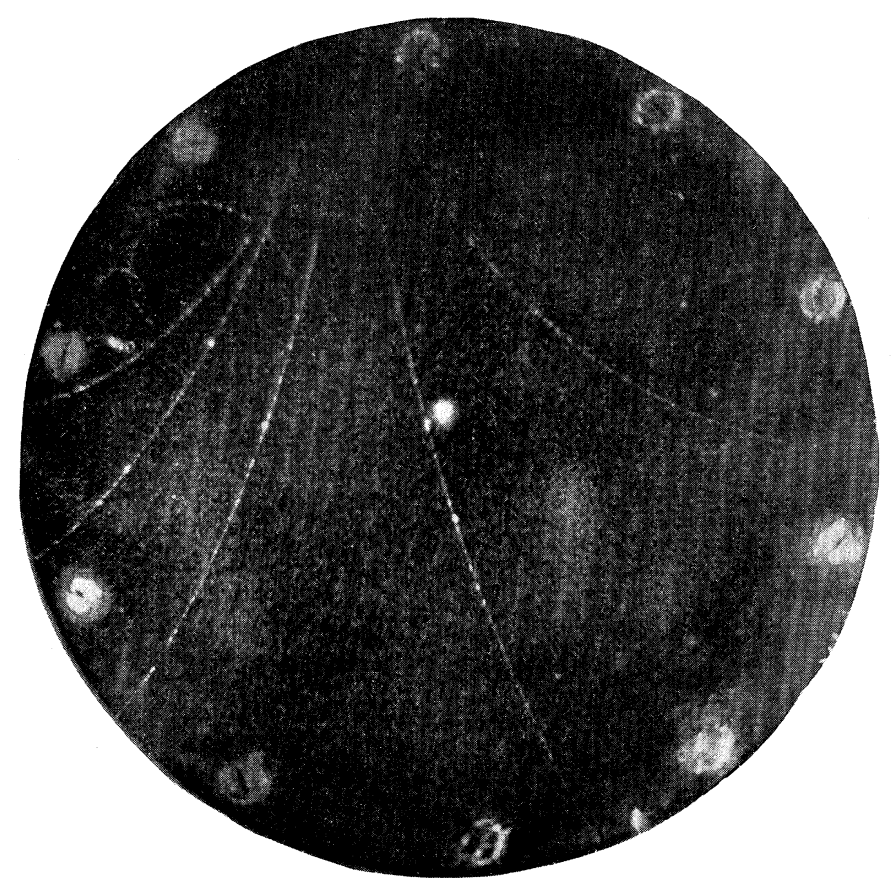

FIG. 8. 17,000 gauss field. A shower presumably originating in the impact of a cosmic-ray photon upon the nucleus of an atom of lead in the bar just above the top counter. Energies in millions of e.v.; positrons, 145, 38, negatrons 104, 65, 28; sum of all 380. Again the presence of secondary photons is demonstrated by the tracks of low energy particles at the left; their energies are: $6.7,4,2,0.1$. 


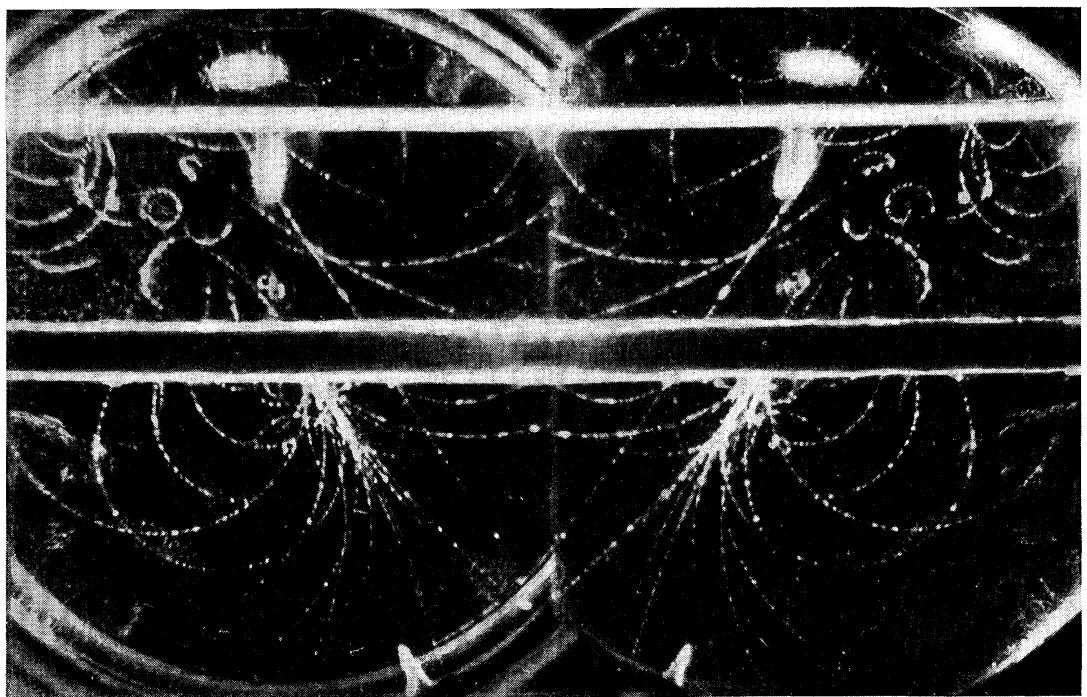

FIG. 9. 17,000 gauss field. The tracks in the upper part of the photograph, at least three of which converge to a region in front of the chamber, indicate the occurrence of a shower above the chamber. A second shower of 22 particles 7 positives, 15 negatives is seen to originate in the lead plate, the initial directions of the particles indicating that the photon (or photons) producing it passed through a point very close to the origin of the upper shower. The high energy positron (520 million electron-volts) passing through both lead plates probably has its origin at the point above the chamber through which passed the photon which gave rise to the showers. There are other tracks not in line with the main shower, as, for example, a group of three tracks from the upper lead plate at the left, which we attribute to the absorption there of secondary photons. The two heavy white patches just above and just below the upper lead plate cannot be associated in time with the shower. Their diffuse appearance may be explained by the assumption that they are due to recoil nuclei released before the expansion. The total energy of all the tracks is about a billion volts. All this suggests that a high energy photon may knock out one or many electrons from several nuclei which it may encounter along its path.

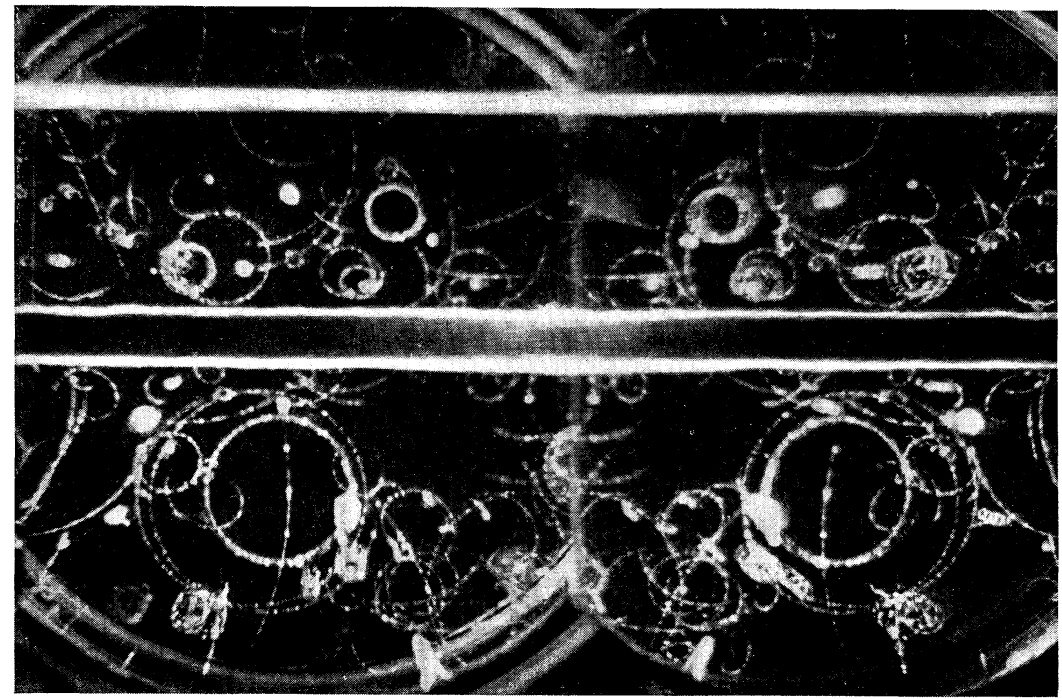

FIG. 10. 17,000 gauss field. More than 80 low energy tracks. A stereoscopic study of the orientations and directions of these tracks shows that in most instances their motions are nearly in the plane of the chamber so they could not have originated except from a considerable number of separate centers, hence indicating a large number of secondary low energy photons $(100,000$ to 10 million electron-volts) presumably resulting from the collision of a primary photon with a lead nucleus above the upper counter. It is this shower that must set off both counters, as well as produce the cloud-chamber effects. 


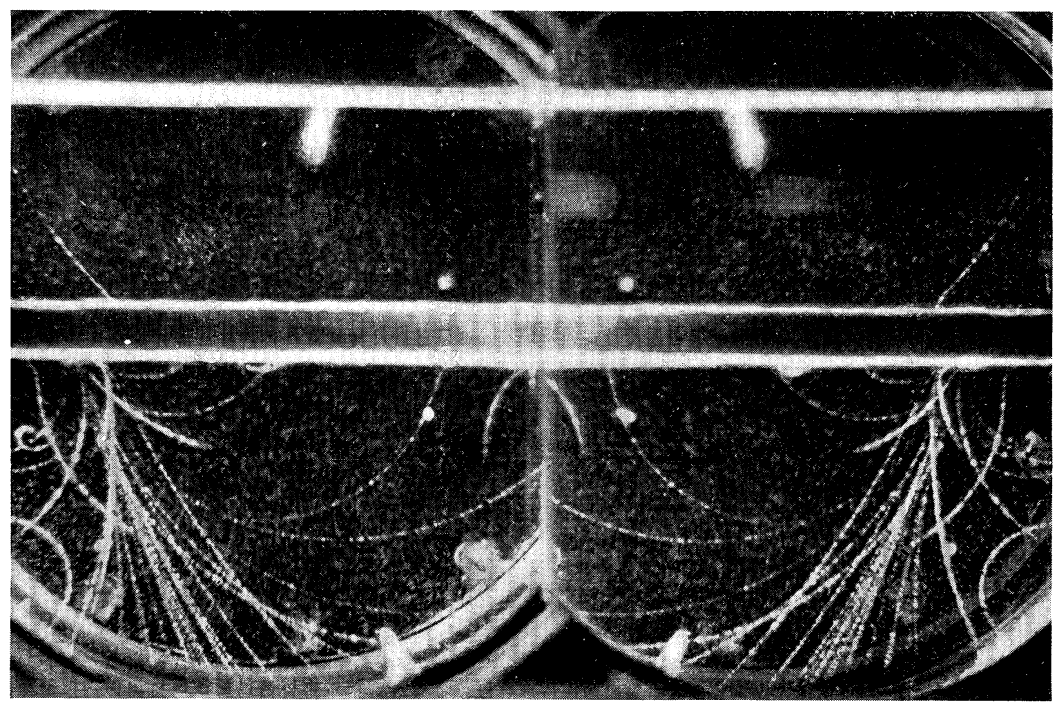

FIG. 11. 17,000 gauss field. Shower of 28 electron tracks resulting presumably from the absorption of a very high energy primary photon in the central lead bar. From one main center at the left there diverge 15 positrons and 10 negatrons, while the three remaining tracks may arise from the photon spray. The total energy is about $2.5 \times 10^{9}$ volts, slightly less than that of the highest energy single tracks we have observed.

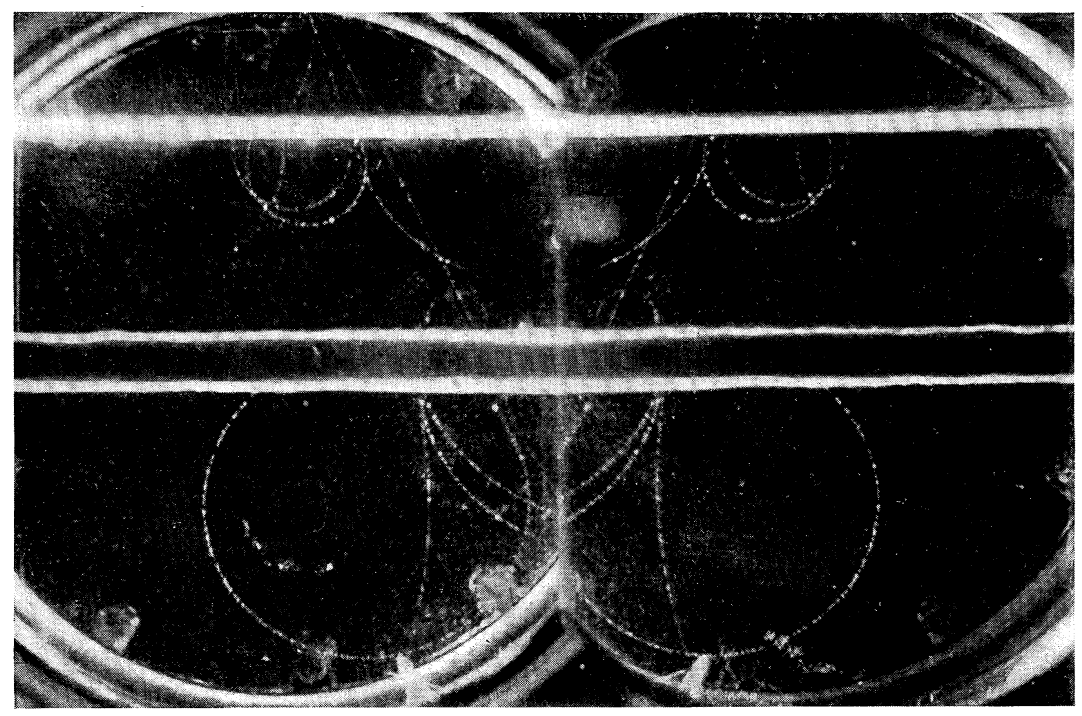

FIG. 12. 17,000 gauss field. An example, like Fig. 9, of associated showers. A pair from the upper sheet of lead and a group of three from the lower are ejected in the same general direction along the same straight line, and the pair which enters the top of the chamber originates not far from the line joining the centers of the two foregoing showers. 

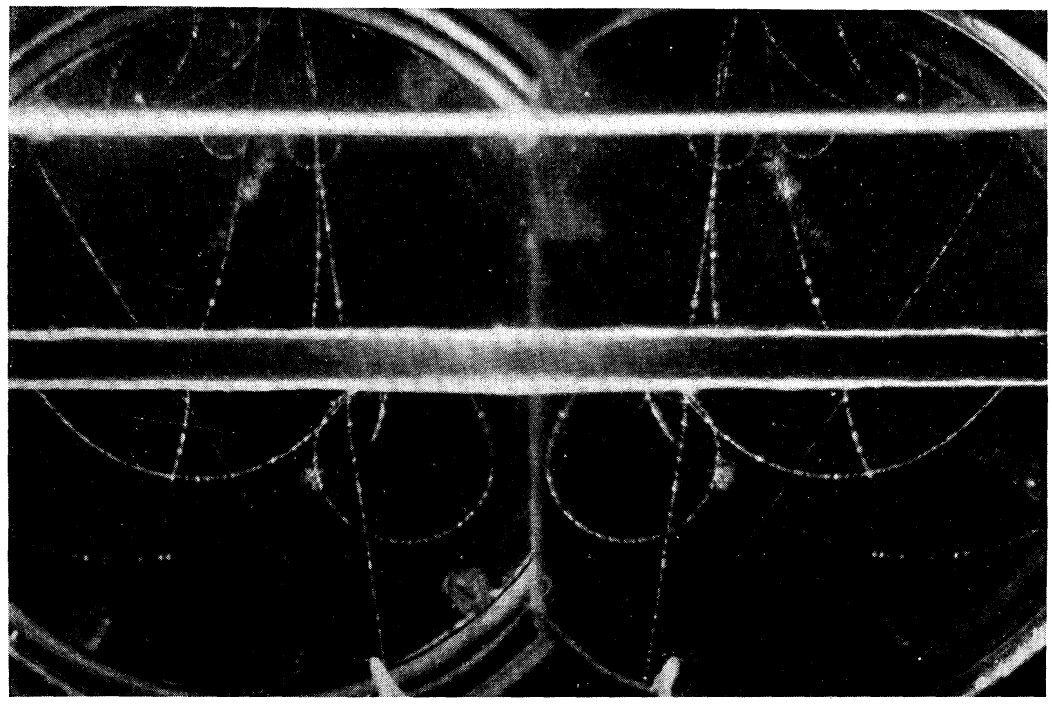

FIG. 13. 17,000 gauss field. One of the two observed cases of formation of a pair by a charged particle. Energies: (-) 16; (+) 7.6 .

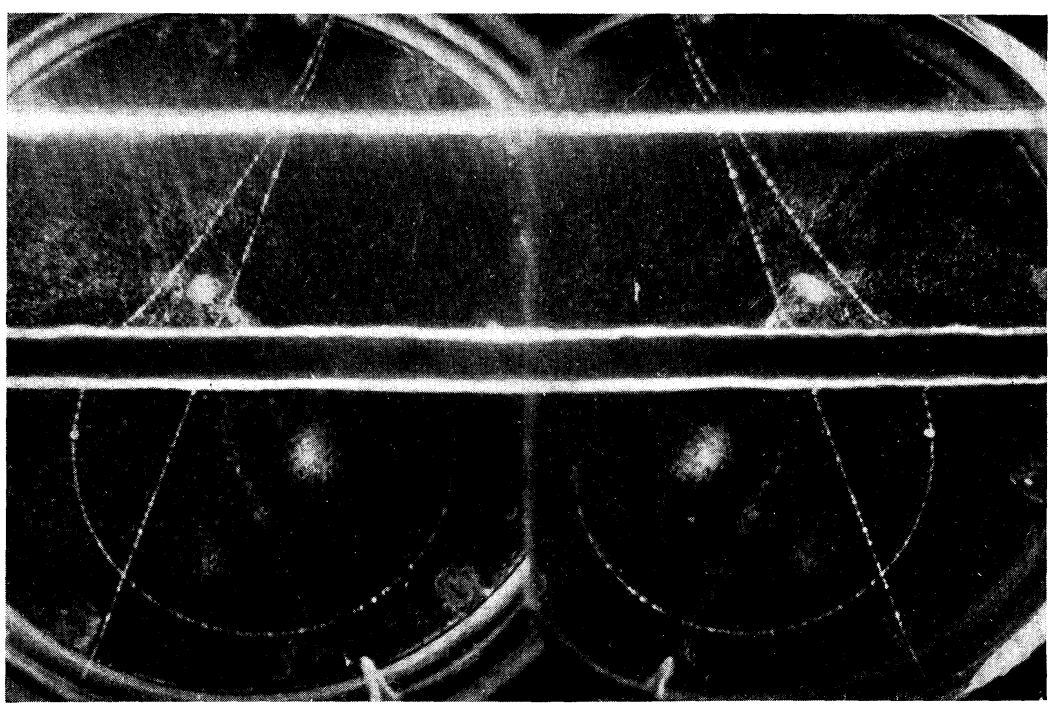

FIG. 14. 17,000 gauss field. At the left an electron passes into the middle lead plate and either transfers its energy to a positron or else forms a pair, both the negatron-component of the pair and the original negatron being absorbed in the lead. The former interpretation seems more likely. The difference in energy above and below the plate is consistent with observed values of the specific energy loss for electrons in lead, inasmuch as the fluctuations are rather large. Energies: (-) above, 90; (+) below, 26. (Specific energy loss $49 \times 10^{6}$ e.v. $/ \mathrm{cm}$. ) 


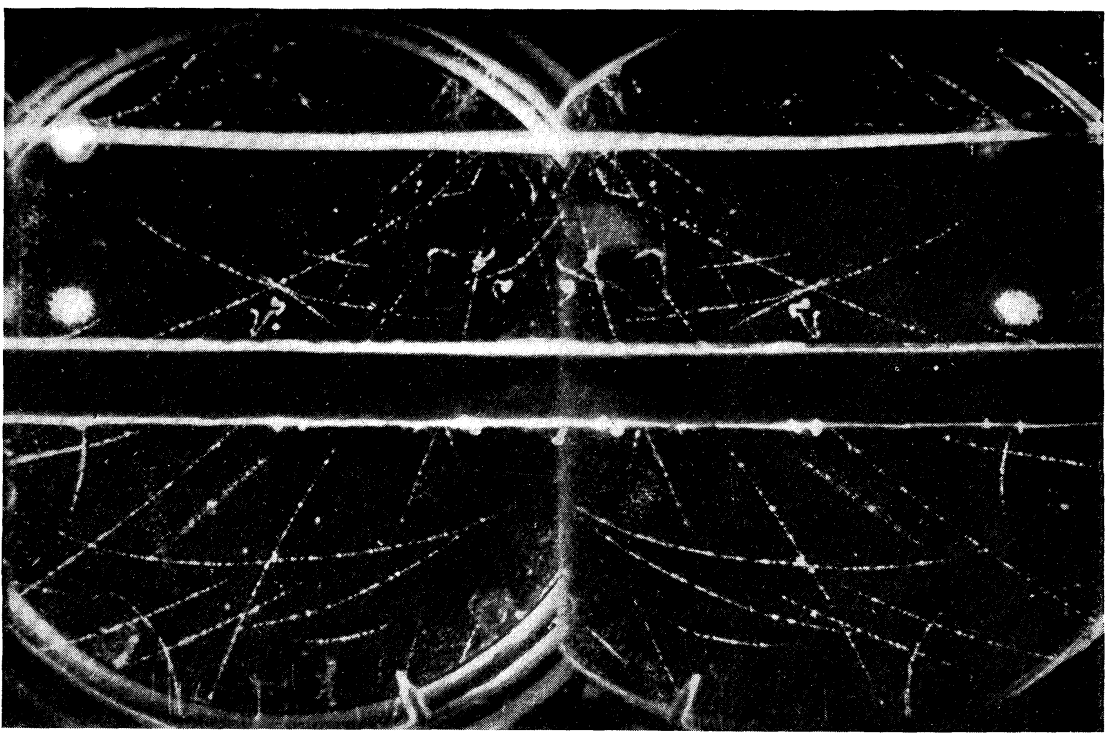

FIG. 15. 17,000 gauss field. The upper plate is lead, $2 \mathrm{~mm}$ thick; the center one, carbon $1.4 \mathrm{~cm}$ thick. A shower occurs in the upper right-hand part of the chamber, sending particles downward into the central slab of carbon. The absence of a shower from the carbon in this photograph, as well as in several other similar ones in which a second shower should certainly have been observed had the carbon been replaced by lead, demonstrates the relative inability of a carbon nucleus to absorb a photon by shower formation. There are, however, indications of extranuclear absorption of low energy photons generated in the shower.

by a multitude of separate particles all accurately associated in time as shown by identity in the sharpness or diffuseness of the tracks, but in general of low energy as revealed by the very strong curvatures of the tracks, since the field is here, for the first time, strong enough when the counter controlled method is used, to produce measureable curvatures in all the tracks. A common characteristic of these photographs is the wide and irregular distribution of the tracks, presumably due to a secondary photon spray (see (4) below).

(4) The fourth result is that there is clear photographic evidence that by a photon encounter with a nucleus in the lead or other solid matter above the upper counter, there is sometimes produced not only a shower of positrons and negatrons, but also a copious spray of relatively soft gamma-rays or photons presumably arising, precisely as in the case of the general gammaradiation resulting from cathode-ray impacts upon the anticathode, from the impact of the electrons $(+$ and -$)$ against the heavy parts of the exceedingly dense nucleus as these electrons, energized by the colliding photon, strive to escape from that nucleus. That these are not in general fluorescence gamma-rays is shown by the fact that these photons in general, though not always, appear below the point of encounter of the primary photon with a nucleus, not above it. Millikan and Neher have proved this by taking an electroscope to high altitudes in an airplane and finding that the ionization in that electroscope is essentially the same whether the electroscope rests upon a very heavy mass of lead or upon a very thin wooden support. The photographs, however, seem to furnish good evidence that occasionally a gamma-ray photon as well as an electron, shoots upward from the point of collision as well as downward. That the foregoing spray consists of gamma-rays or photons, rather than of neutrons, is proved by the fact that, in being absorbed by the surrounding matter, this spray apparently throws out many extranuclear electrons with energies from below 100,000 volts up to 20 or 30 million electron-volts-a behavior foreign to the nature of neutrons, which can impart high energies only to bodies of nuclear mass. None of our photographs taken thus far with this apparatus reveal the tracks of protons or of heavier nuclei, associated in time with the tracks 
of the shower. We have, however, two or three isolated tracks which seem to be those of recoil nuclei. ${ }^{6}$ These results are particularly evident from the photographs 7 to 15 . Our interpretation of these photographs is set forth in the captions.

(5) The fifth result is that when no lead is inserted in the middle of the cloud chamber, out of 145 track photographs 141 show one track that at least may go through both counters, while 4 show no apparent possibility of a single track traversing both, but do show the characteristic photon-spray tracks. When, however, the strip of lead one centimeter thick is placed across the middle of the cloud chamber, out of 397 track photographs there are 358 in which one track may go through both counters, while there are 39 cases in which both counters are definitely set off by low energy sprays. In other words, $4 / 145$ or 2.8 percent of the coincidences obtained with no intervening lead, do not represent a track through both counters. While when one centimeter of lead intervenes between the counters, $39 / 397$ or 9.8 percent do not represent one single track going straight through both counters. It is then most significant for the theory of counter action that a piece of lead only one centimeter thick, between the counters, increases the number of coincidences which do not represent a straight track, from 2.8 to 9.8 percent. For thick lead plates between the counters, the differences would of course be higher. Here is, then, very direct photographic evidence for the correctness of the foregoing theory of counter action when large amounts of heavy matter intervene between the counters.

(6) The sixth result of these studies of counteraction is that when a slab of carbon, $1.4 \mathrm{~cm}$ thick, replaces the slab of lead in the middle of the chamber (see Fig. 15), very few showers or sprays appear, and as yet no evidence of neutron

\footnotetext{
${ }^{6}$ While diffuse patches of ionization such as those reported by Locher (Phys. Rev. 44, 779, 1933) occasionally appear in our photographs, it does not seem certain that they represent nucleus tracks produced by neutron encounters as he suggests. In fact, such patches of ionization occur more frequently immediately after the chamber has been filled with fresh air, and at least a part of their number therefore cannot represent cosmic-ray effects.
}

encounters. Out of 441 successful track photographs taken this way, while they show many showers coming in from above, nine of which have more than five tracks, there is no evidence of any secondary centers whatever formed within the carbon by secondary photons. There are in this case 29 photographs which show no apparent possibility of a single track traversing both counters, but do show the characteristic photon spray tracks, as compared to 412 cases where one track did pass through both counters; in 6.6 percent of the cases, therefore, a photon spray was responsible for the simultaneous responses.

(7) The seventh result of these studies is to show that when a shower occurs, the total energy in the shower is thus far never higher than the energy appearing in single electron shots. In other words, the energy of the incident photon may apparently be largely transferred to a single positron or negatron, or it may be divided between many such, and the accompanying spray of gamma-ray photons. Since 88 percent of the photographs show single electron shots even when materials of large atomic weight surround the chamber, the production of a shower is apparently a relatively rare event.

(8) The eighth result of these studies is to indicate that the simplest interpretation of the nature of the interaction of cosmic rays with the nuclei of atoms, lies in the assumption that when a cosmicray photon impinges upon a heavy nucleus, electrons of both signs are ejected from that nucleus and appear in the form of the positrons and negatrons shown in our photographs. The large, and the, in general uneven number of positrons and negatrons appearing in such photographs as those shown in Figs. 9 and 11, for example, seem difficult to reconcile with the Dirac theory, as interpreted by Blackett and Occhialini, of the creation of electron-pairs out of the incident photons, and point strongly to the existence of nuclear reactions of a type in which the nucleus plays a more active rôle than merely that of a catalyst. The essential difference between the two points of view is that in one case the nucleus changes its character and in the other it does not do so. 


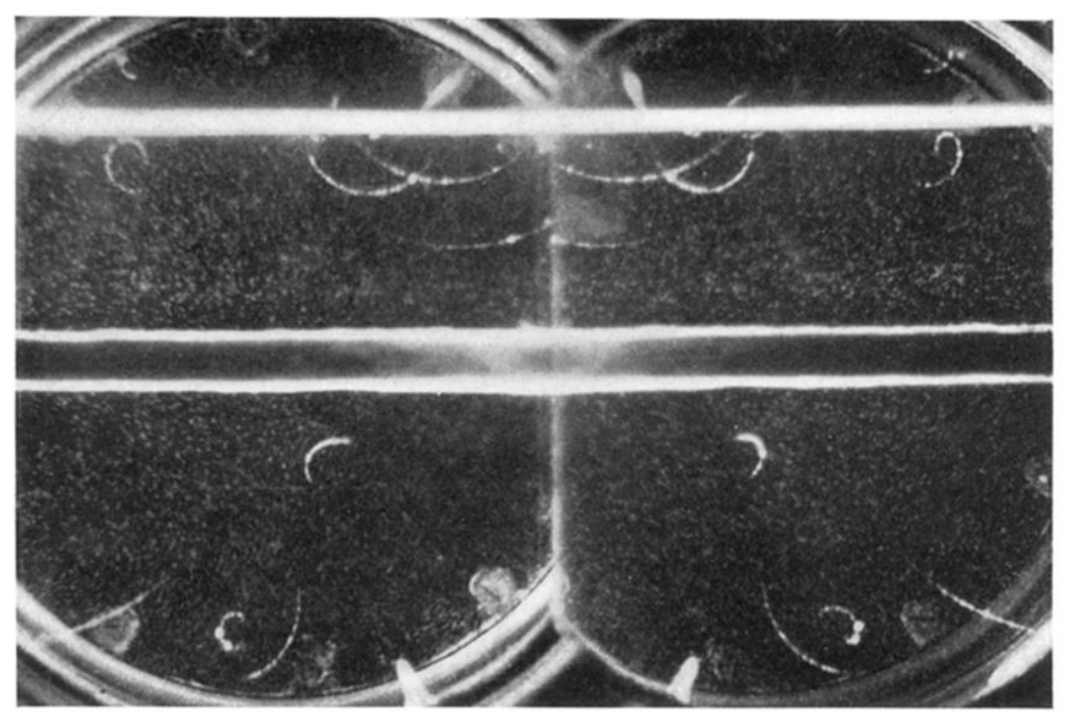

FIG. 1. 17,000 gauss field. The left-hand exposure is the direct photograph, the other the reflection taken for stereoscopic purposes. Six low energy electrons are seen between the two lead plates and four below the lower one. Stereoscopic vision shows that eight of these ten come from the wall and two from the upper lead plate. The short, very heavy track above the top plate, probably not associated in time with the others, may be a recoil nucleus. 

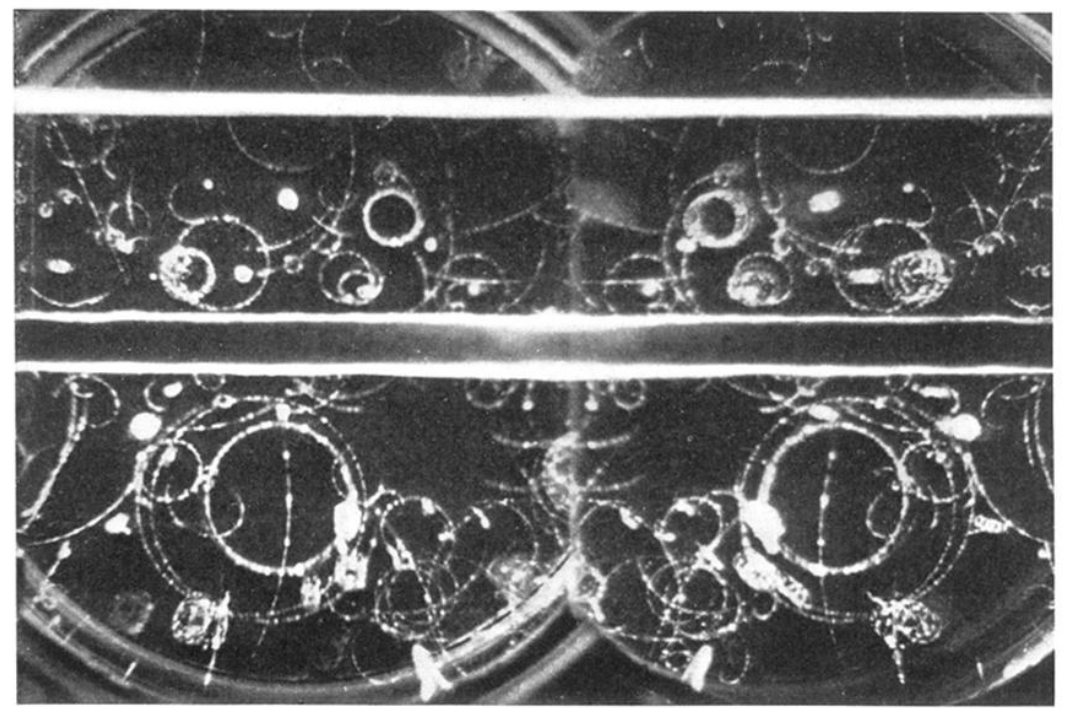

Fig. 10. 17,000 gauss field. More than 80 low energy tracks. A stereoscopic study of the orientations and directions of these tracks shows that in most instances their motions are nearly in the plane of the chamber so they could not have originated except from a considerable number of separate centers, hence indicating a large number of secondary low energy photons $(100,000$ to 10 million electron-volts) presumably resulting from the collision of a primary photon with a lead nucleus above the upper counter. It is this shower that must set off both counters, as well as produce the cloud-chamber effects. 


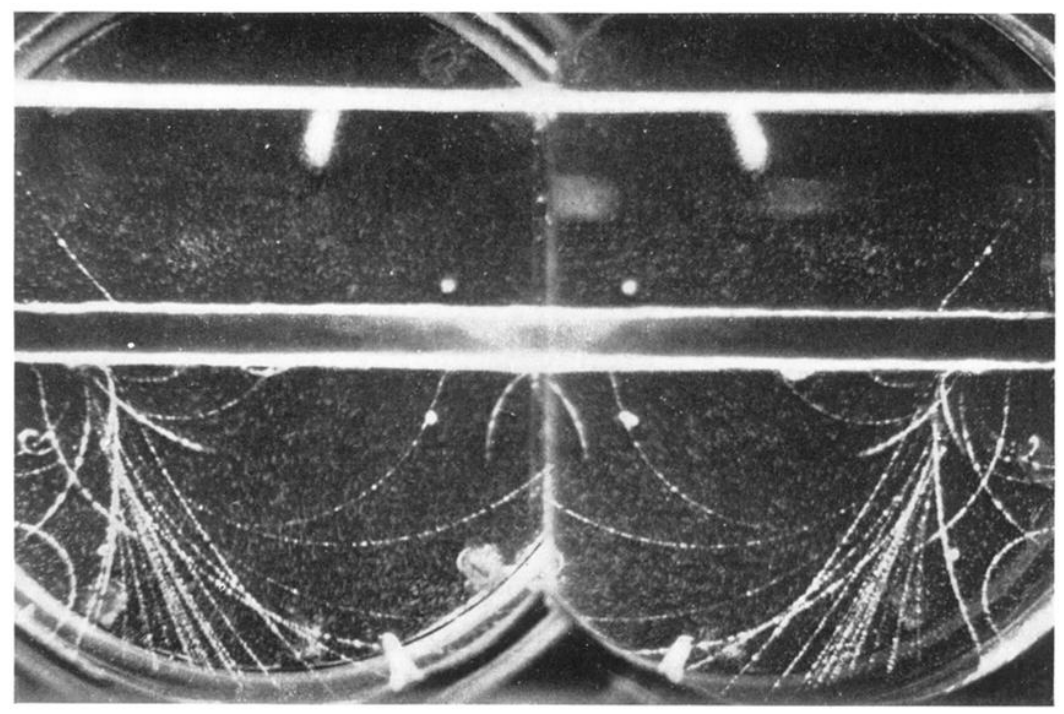

FIG. 11. 17,000 gauss field. Shower of 28 electron tracks resulting presumably from the absorption of a very high energy primary photon in the central lead bar. From one main center at the left there diverge 15 positrons and 10 negatrons, while the three remaining tracks may arise from the photon spray. The total energy is about $2.5 \times 10^{9}$ volts, slightly less than that of the highest energy single tracks we have observed. 


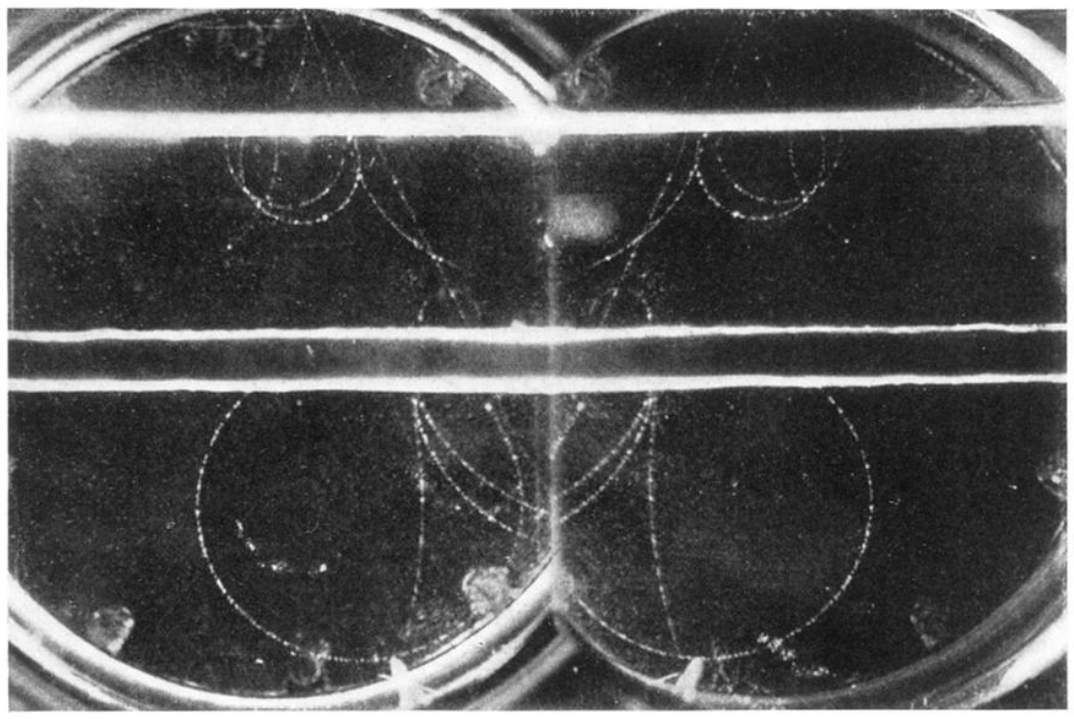

FIG. 12. 17,000 gauss field. An example, like Fig. 9, of associated showers. A pair from the upper sheet of lead and a group of three from the lower are ejected in the same general direction along the same straight line, and the pair which enters the top of the chamber originates not far from the line joining the centers of the two foregoing showers. 


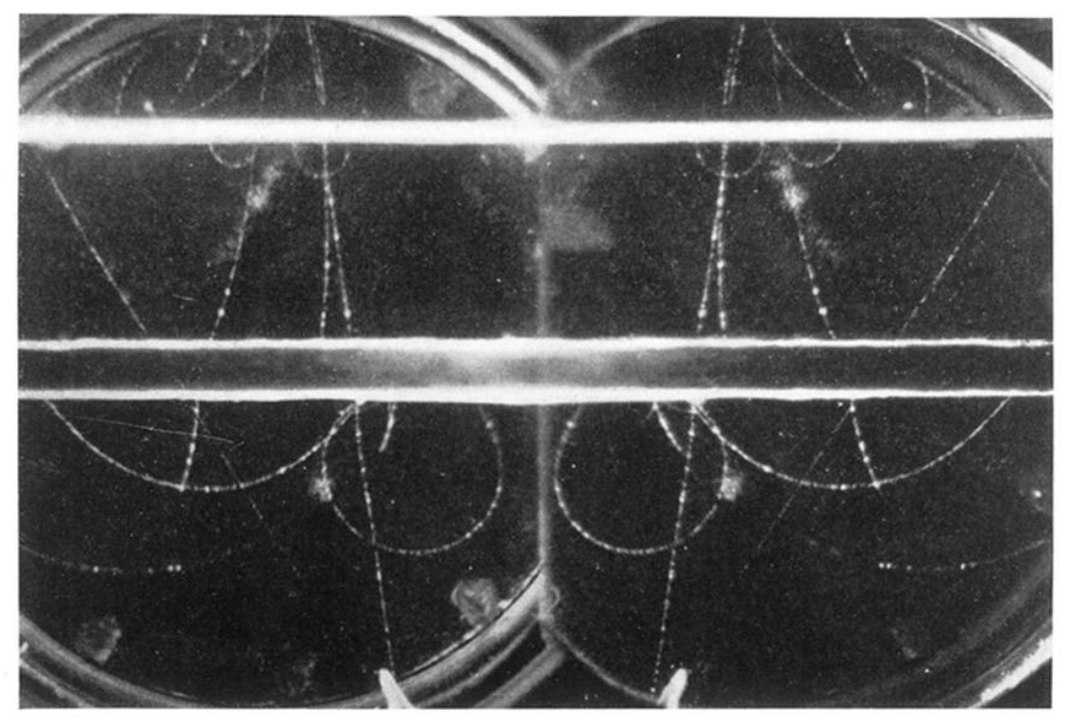

FIG. 13. 17,000 gauss field. One of the two observed cases of formation of a pair by a charged particle. Energies: (-) 16; (+) 7.6. 


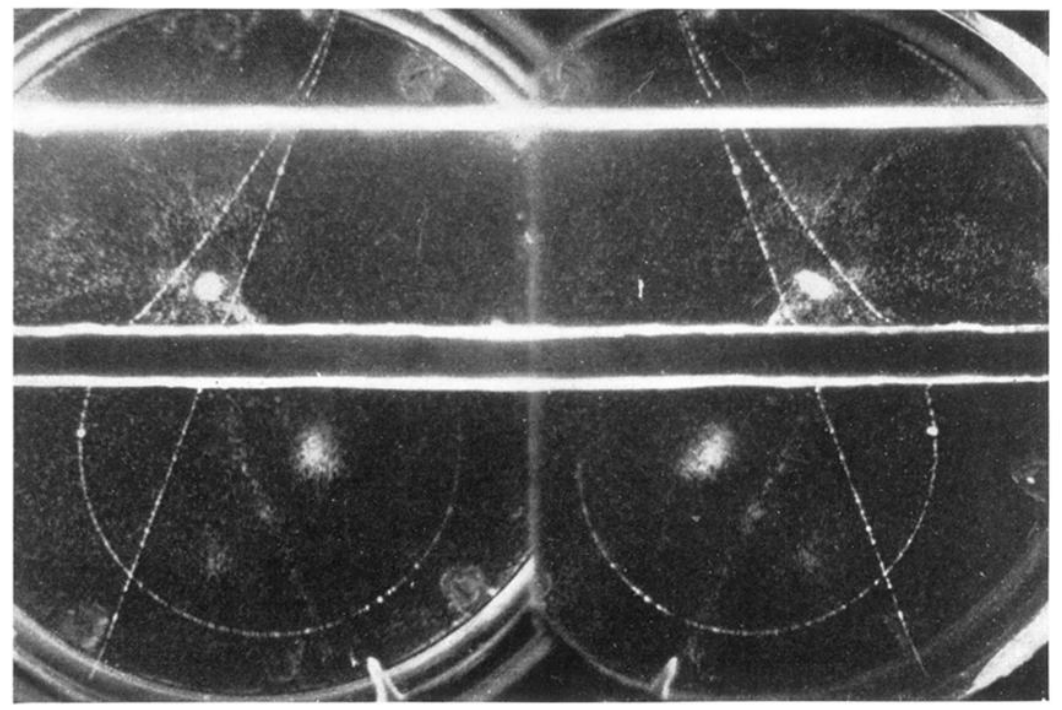

FIG. 14. 17,000 gauss field. At the left an electron passes into the middle lead plate and either transfers its energy to a positron or else forms a pair, both the negatron-component of the pair and the original negatron being absorbed in the lead. The former interpretation seems more likely. The difference in energy above and below the plate is consistent with observed values of the specific energy loss for electrons in lead, inasmuch as the fluctuations are rather large. Energies: $(-)$ above, $90 ;(+)$ below, 26. (Specific energy loss $49 \times 10^{6}$ e.v. $/ \mathrm{cm}$.) 


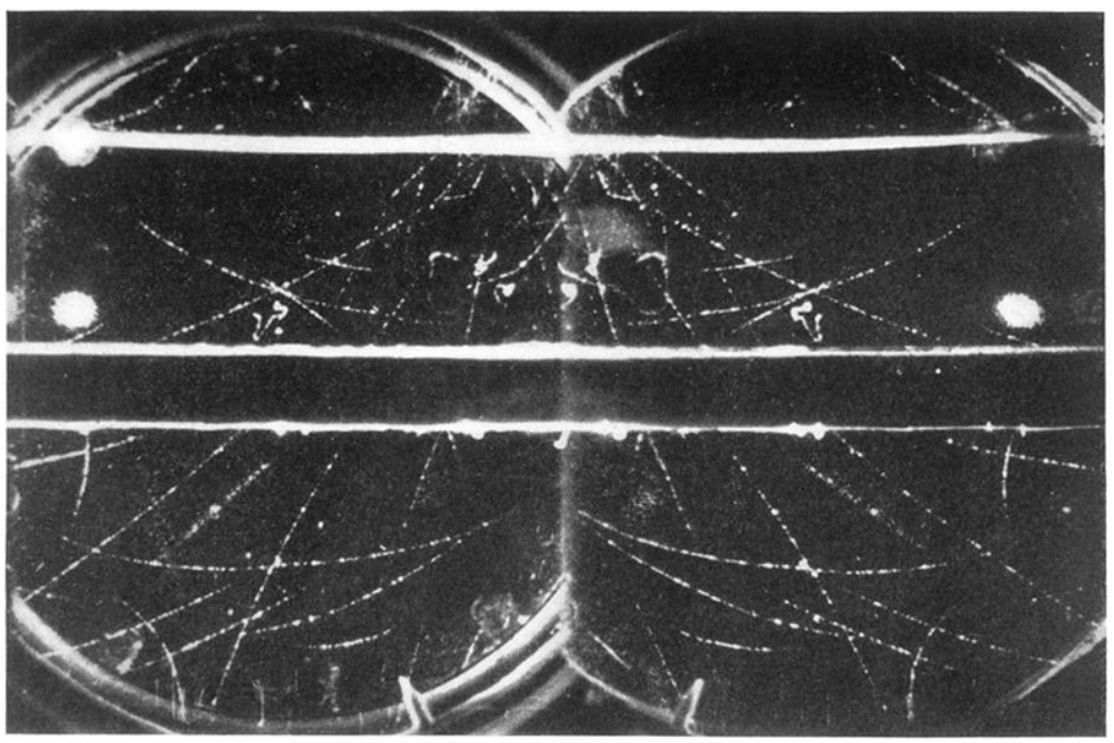

FIG. 15. 17,000 gauss field. The upper plate is lead, $2 \mathrm{~mm}$ thick; the center one, carbon $1.4 \mathrm{~cm}$ thick. A shower occurs in the upper right-hand part of the chamber, sending particles downward into the central slab of carbon. The absence of a shower from the carbon in this photograph, as well as in several other similar ones in which a second shower should certainly have been observed had the carbon been replaced by lead, demonstrates the relative inability of a carbon nucleus to absorb a photon by shower formation. There are, however, indications of extranuclear absorption of low energy photons generated in the shower. 


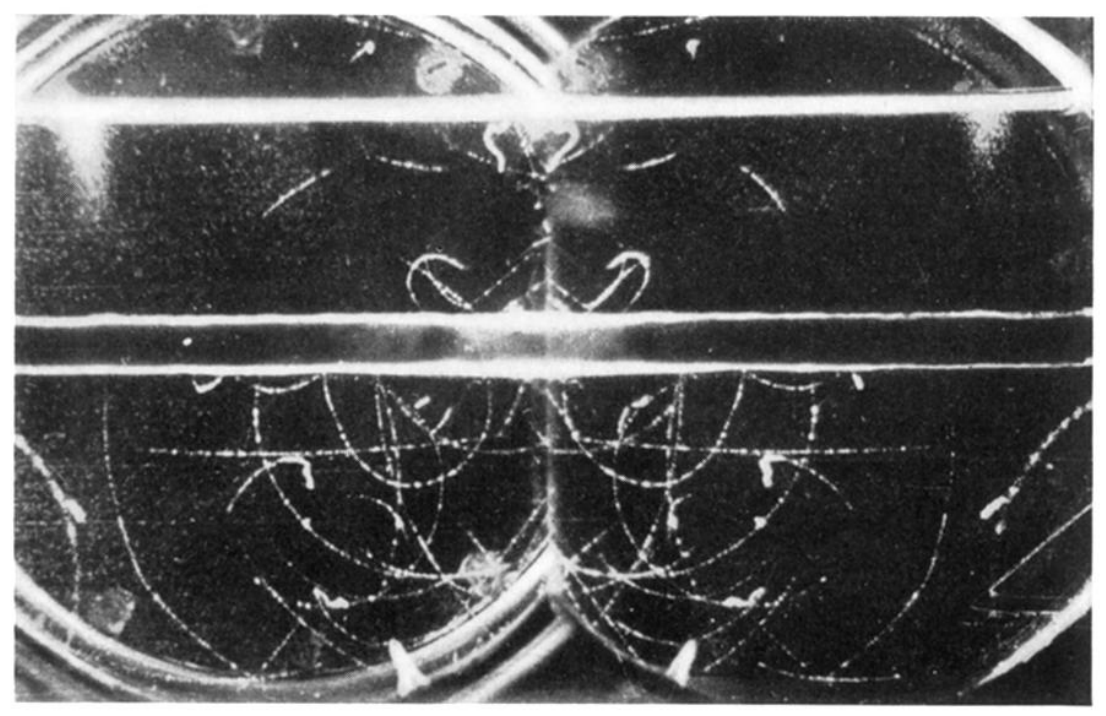

FIG. 2. 825 gauss field. This photograph shows eight electron tracks between the two plates and 15-20 below the central one, mostly of the order of but a million electron-volts. Stereoscopic vision shows two pairs produced within the central plate and emerging from its lower face. Most of the remaining electrons come from the walls. Their distribution in direction is extremely various, indicating that they arise from the absorption of a spray of photons, probably originating in the lead bar above the upper counter. The horizontal track cutting across all the others is particularly interesting. 


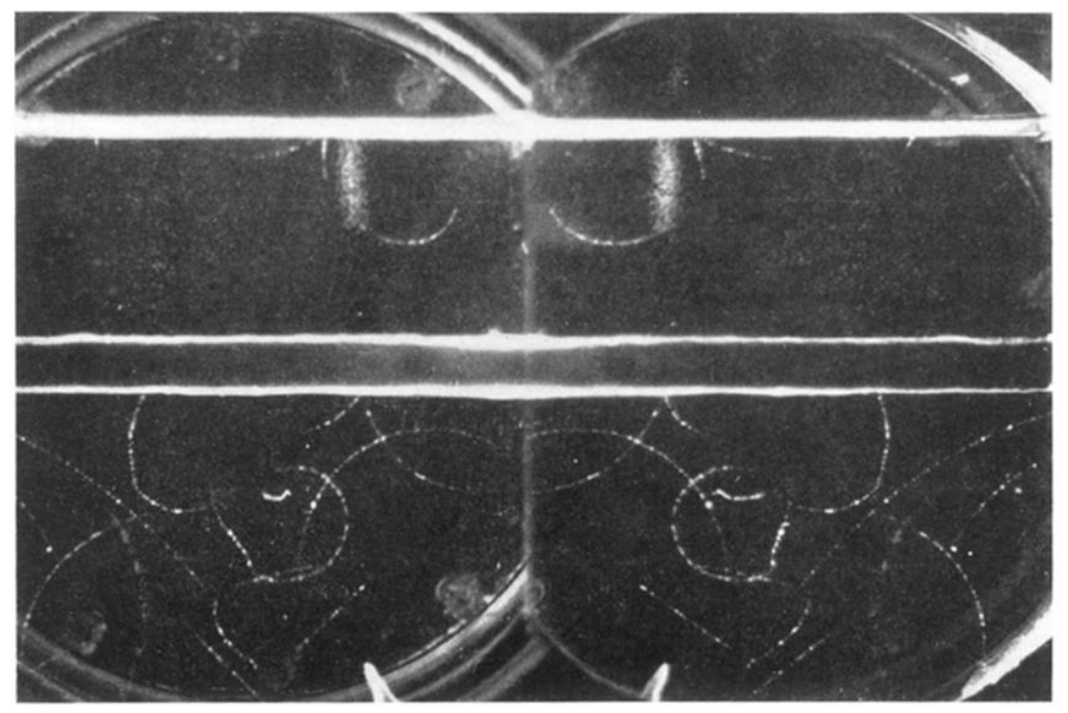

FIG. 3. 825 gauss field. A pair ejected from the upper lead plate and another from the central lead plate; also a group of some fifteen tracks of irregular distribution all ejected by soft photon rays, probably originating in the lead bar above the upper counter. 

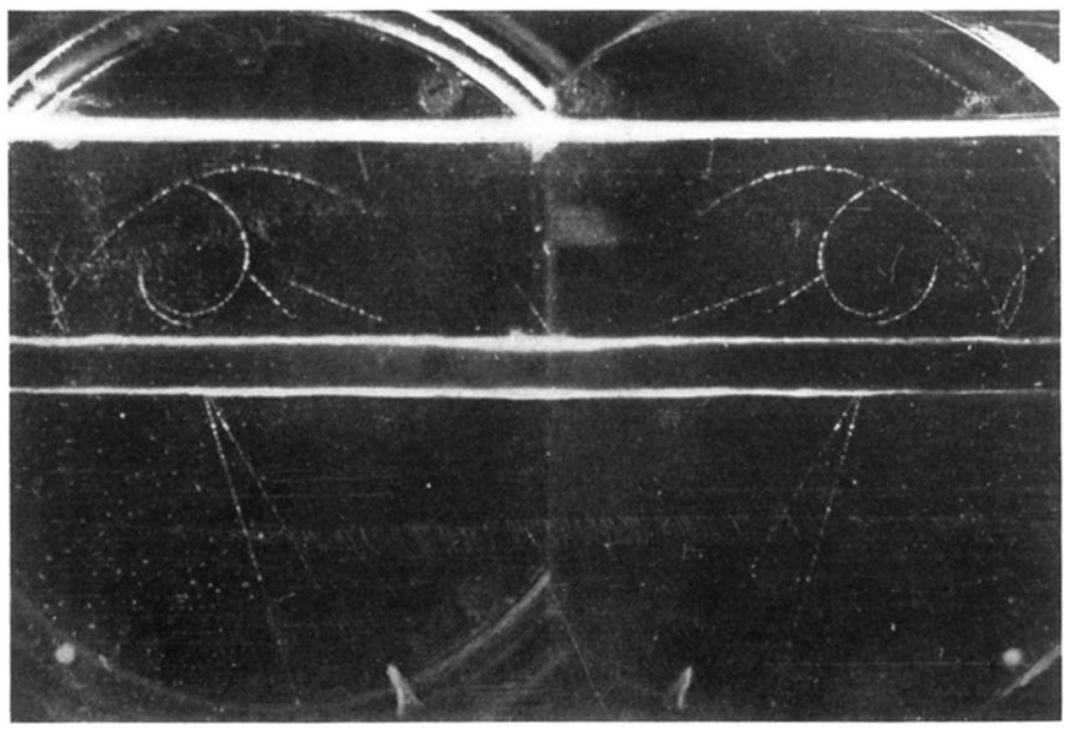

FIG. 4. 825 gauss field. A rather high energy pair is ejected from the lead plate by the absorption within it of a photon. The signs and energies of the components cannot be determined because of the weakness of the field. 

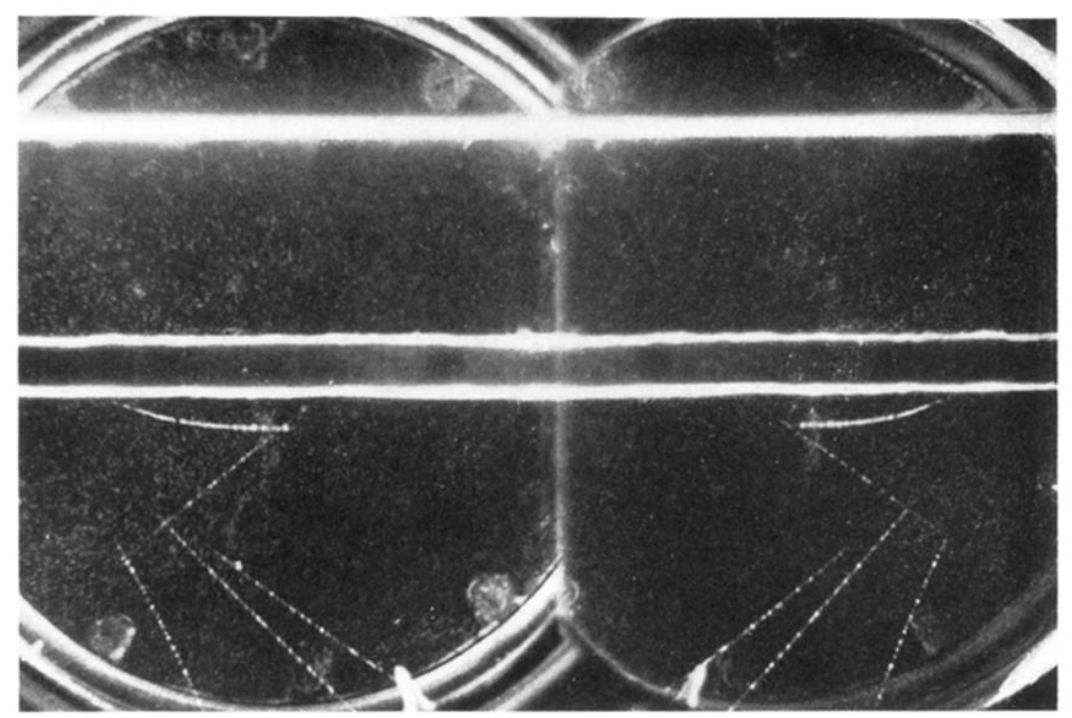

FIG. 5. 17,000 gauss field. Four tracks come from a common region behind the chamber. The fifth is definitely associated with them in time but has a totally different origin. We again postulate a secondary photon spray. 


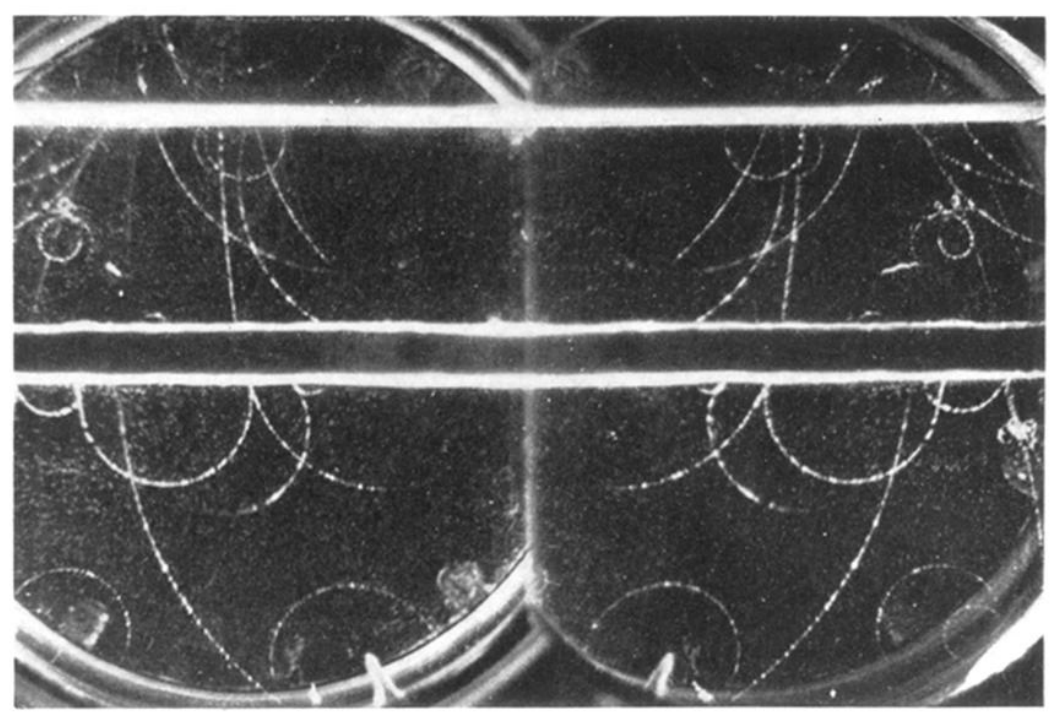

Fig. 6. 17,000 gauss field. Here again no single particle goes through the counters and the cloud chamber, but a secondary photon spray originating above ejects from the lead plates and the walls several pairs and a number of single electrons. A positron of energy $140 \times 10^{6}$ electronvolts penetrates the central $1 \mathrm{~cm}$ lead plate and emerges with an energy of $13 \times 10^{6}$ electronvolts after giving by a close encounter $6.7 \times 10^{6}$ electron-volts to a negatron. 


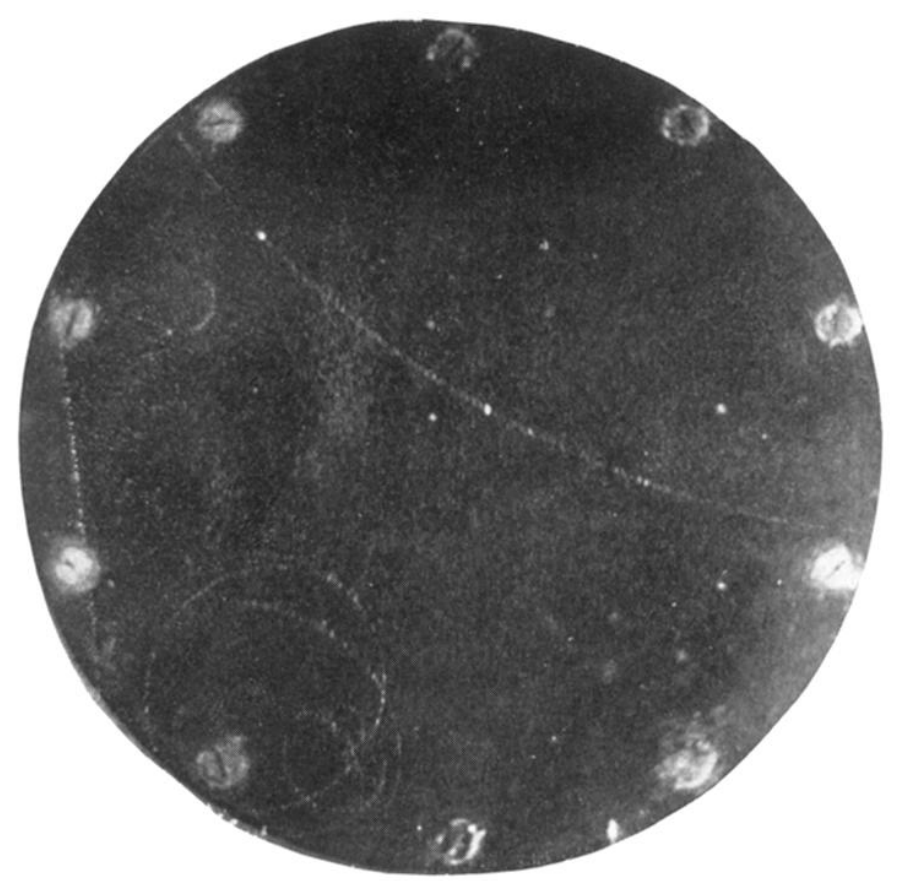

FIG. 7. 17,000 gauss field. An electron pair, positron 75 million electron-volts, negatron 290 million ejected presumably from the nucleus of a lead atom above the upper counter. These electrons in getting through or out of the nucleus presumably collided with its mass, and produced thereby "brems-strahlung." This photon spray shot downward and its absorption in the gas of the chamber, or the surface layer of its wall, produced the four secondaries seen between the electron tracks of energies in millions of electron-volts 9, 9, 4, 1 . 


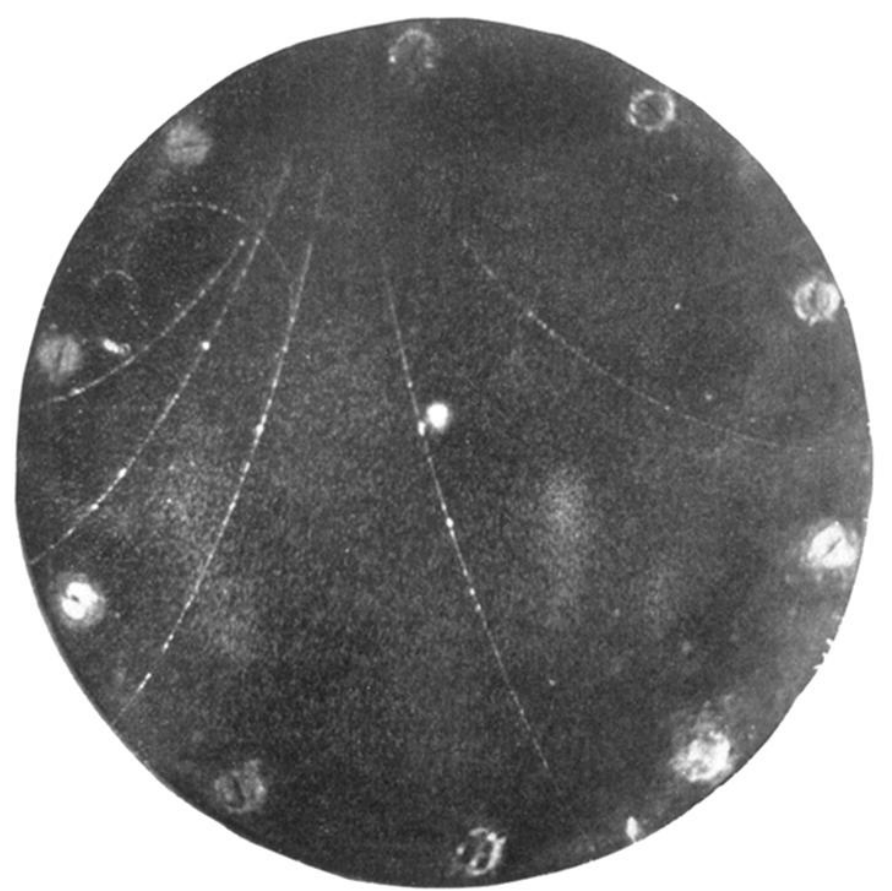

FIG. 8. 17,000 gauss field. A shower presumably originating in the impact of a cosmic-ray photon upon the nucleus of an atom of lead in the bar just above the top counter. Energies in millions of e.v.; positrons, 145, 38, negatrons 104, 65, 28; sum of all 380. Again the presence of secondary photons is demonstrated by the tracks of low energy particles at the left; their energies are: $6.7,4,2,0.1$. 


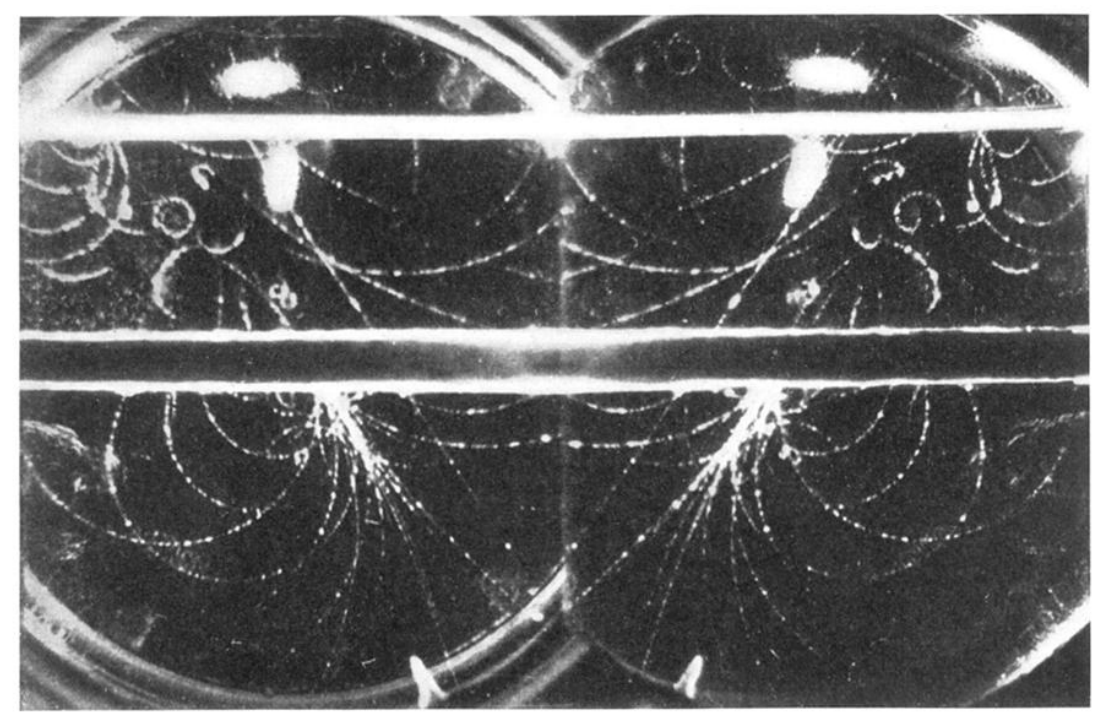

FIG. 9. 17,000 gauss field. The tracks in the upper part of the photograph, at least three of which converge to a region in front of the chamber, indicate the occurrence of a shower above the chamber. A second shower of 22 particles 7 positives, 15 negatives is seen to originate in the lead plate, the initial directions of the particles indicating that the photon (or photons) producing it passed through a point very close to the origin of the upper shower. The high energy positron (520 million electron-volts) passing through both lead plates probably has its origin at the point above the chamber through which passed the photon which gave rise to the showers. There are other tracks not in line with the main shower, as, for example, a group of three tracks from the upper lead plate at the left, which we attribute to the absorption there of secondary photons. The two heavy white patches just above and just below the upper lead plate cannot be associated in time with the shower. Their diffuse appearance may be explained by the assumption that they are due to recoil nuclei released before the expansion. The total energy of all the tracks is about a billion volts. All this suggests that a high energy photon may knock out one or many electrons from several nuclei which it may encounter along its path. 